\title{
Periodontal treatment during pregnancy decreases the rate of adverse pregnancy outcome: a controlled clinical trial
}

\author{
Adriana Campos Passanezi SANT'ANA 1 , Marinele R. de CAMPOS ${ }^{2}$, Selma Campos PASSANEZI ${ }^{3}$, \\ Maria Lúcia Rubo de REZENDE ${ }^{1}$, Sebastião Luiz Aguiar GREGHI ${ }^{4}$, Euloir PASSANEZI ${ }^{5}$ \\ 1- DDS, PhD, Associate Professor, Department of Prosthodontics, Bauru School of Dentistry, University of São Paulo, Bauru, SP, Brazil.
2- DDS, PhD, Clinical Professor, University of Southern California, Los Angeles, CA, USA.
3- DDS, Tutor of Extracurricular Activities, Department of Pediatric Dentistry, Orthodontics and Community Health, Bauru School of Dentistry, University of
São Paulo; Dentist, Public Health Service, Bauru, SP, Brazil.
4- DDS, PhD, Assistant Professor, Department of Prosthodontics, Bauru School of Dentistry, University of São Paulo, Bauru, SP, Brazil.
5- DDS, PhD, Professor, Department of Prosthodontics, Bauru School of Dentistry, University of São Paulo, Bauru, SP, Brazil.
}

Corresponding address: Profa. Adriana Campos Passanezi Sant'Ana - Faculdade de Odontologia de Bauru - USP - Departamento de Prótese - Disciplina de Periodontia - Al. Otávio Pinheiro Brisolla, 9-75 - 17012-901 - Bauru - SP - Brazil - Phone: +55-14-3235-8278 - Fax: +55-14-3227-5105 - e-mail: acpsantana@usp.br

Received: June 13, 2009 - Modification: February 16, 2010 - Accepted: February 21, 2010

\section{ABSTRACT}

$\mathrm{O}$ bjectives: The aim of this study was to evaluate the effects of non-surgical treatment of periodontal disease during the second trimester of gestation on adverse pregnancy outcomes. Material and Methods: Pregnant patients during the $1^{\text {st }}$ and $2^{\text {nd }}$ trimesters at antenatal care in a Public Health Center were divided into 2 groups: NIG - "no intervention" $(n=17)$ or IG- "intervention" $(n=16)$. IG patients were submitted to a non-surgical periodontal treatment performed by a single periodontist consisting of scaling and root planning (SRP), professional prophylaxis (PROPH) and oral hygiene instruction (OHI). NIG received PROPH and OHI during pregnancy and were referred for treatment after delivery. Periodontal evaluation was performed by a single trained examiner, blinded to periodontal treatment, according to probing depth (PD), clinical attachment level (CAL), plaque index (PI) and sulcular bleeding index (SBI) at baseline and 35 gestational weeks-28 days post-partum. Primary adverse pregnancy outcomes were preterm birth ( $<37$ weeks), low birth weight $(<2.5 \mathrm{~kg})$, late abortion $(14-24$ weeks) or abortion ( $<14$ weeks). The results obtained were statistically evaluated according to OR, unpaired $t$ test and paired $t$ test at $5 \%$ significance level. Results: No significant differences were observed between groups at baseline examination. Periodontal treatment resulted in stabilization of CAL and PI $(p>0.05)$ at IG and worsening of all periodontal parameters at NIG $(p<0.0001)$, except for PI. Significant differences in periodontal conditions of IG and NIG were observed at 2 nd examination $(p<0.001)$. The rate of adverse pregnancy outcomes was $47.05 \%$ in NIG and $6.25 \%$ in IG. Periodontal treatment during pregnancy was associated to a decreased risk of developing adverse pregnancy outcomes [OR=13.50; CI: $1.47-123.45 ; p=0.02$ ]. Conclusions: Periodontal treatment during the second trimester of gestation contributes to decrease adverse pregnancy outcomes.

Key words: Periodontal diseases. Preterm birth. Low birth weight. Pregnancy. Clinical trials. 


\section{INTRODUCTION}

Infections may play an important role in prematurity ${ }^{11,25}$. After establishing that ascending genitourinary infections are associated to $50 \%$ of preterm birth ${ }^{11}$, other infections remote from fetalplacental unity were regarded as a potential role for preterm birth as well ${ }^{25}$.

Recently, periodontal disease was associated to preterm birth or low birth weight (PLBW) ${ }^{1,6,22,23}$ by bacterial translocation through blood circulation or production of inflammatory mediators $22,23,25$ associated with the onset of delivery ${ }^{17,25,29}$. However some studies observed no influence of periodontal disease on PLBW based on clinical findings ${ }^{7,19,20}$. Most of these were undertaken in low social-educational level patients, and predominantly blacks, who are at increased risk for both periodontal disease and preterm birth ${ }^{3,17}$.

Offenbacher, et al. ${ }^{21}$ (2006) reported that an increased risk of preterm or low birth weight would be related to the progression of periodontal disease during pregnancy or to the prior existence of severe periodontal lesions. These findings confirmed the results of other clinical ${ }^{9}$ and laboratorial studies ${ }^{16}$, suggesting that patients with severe and generalized periodontal disease or progressive disease during pregnancy would be more susceptible to develop adverse pregnancy outcomes.

In an attempt to establish a clear correlation between periodontal disease and PLBW, some studies evaluated the effects of controlling periodontal infection during early phases of pregnancy on pregnancy complications outcome, resulting in a decrease of PLBW $8,10,12,13,18,26$.

The Brazilian Health Department has recently proposed a protocol that should be followed by all public Health Care Units aiming at a better attention to the pregnant and the newborn during ante-natal care and immediately after birth, in order to reduce the high rates of mother and newborn mortality and morbidity 5 . However, oral health was not contemplated in this protocol. In spite of improvement in antenatal care in well developed countries, no significant changes in preterm birth or low birth weight could be observed ${ }^{17}$. Considering that, the aim of this study was to evaluate if the treatment of periodontal disease during early stages of pregnancy would contribute to a reduction in the rate of PLBW in a Brazilian sample population participating of an experimental antenatal health care program that included periodontal treatment and education in oral health.

\section{MATERIAL AND METHODS}

Ethical approval was obtained from the Bauru School of Dentistry-USP Ethics Committee prior to commencement and written informed consent was obtained from all subjects prior to participation (\#102/2002). The clinical trial was registered at Clinicaltrials.gov (NCT 00893802). All pregnant women participating at an Antenatal Care Program at Jardim Redentor I Public Health Center from August 2001 to August 2002 were invited to participate in the study. Inclusion criteria were confirmed singleton gestations between 9 and 24 weeks in healthy women 16-39 years old. Exclusion criteria included non-confirmed pregnancy, age inferior to 16 years or superior to 39 years, multiple gestations, smoking, alcohol or drugs abuse, history of congenital heart disease, current use of corticosteroids or antibiotics, and the presence of systemic conditions that would pose a risk for the mother or the baby, such as diabetes, hypertension, or genitor-urinary infections.

Sample size was initially established at approximately 150 patients, based on the number of pregnant women regularly attending the Public Health Center. However, from this total, 65 patients refused to participate in the study or did not return for baseline examination. From the remaining 85 patients, 52 were at the third trimester and could not be included in the study, as described in Figure 1 , resulting in 33 patients allocated to the different groups.

\section{Obstetrical and maternal data}

Patients filled out a health questionnaire in order to evaluate general health conditions, past and current medical and dental history, and obstetric conditions, in order to control for most of the known risk factors for preterm delivery. Demographic data, such as age, marital status, and educational level, as well as history of previous preterm birth, abortion, late abortion, number of children born alive, and current pregnancy information were recorded. Prenatal care included a minimum of 6 visits during pregnancy up to delivery, blood pressure measurements, dietary consultation, evaluation of weight gain, laboratorial tests to investigate anemia, white blood cell and platelet counting, infectious diseases (e.g., HIV, tuberculosis), urine tests and ultra-sound examinations performed at the $12^{\text {th }}, 20^{\text {th }}$ and $32^{\text {nd }}$ weeks of gestation.

\section{Study design}

All patients $(n=33)$ entering the study at the $1^{\text {st }}$ and $2^{\text {nd }}$ trimesters were invited to participate in an oral health program included in the ante-natal care program. This oral health program included community lectures to inform the mother about oral diseases, such as caries lesions and periodontal disease, and the possible role of periodontal disease in preterm birth and low birth weight. Other measures included personal oral hygiene 
instruction, caries diagnosis and treatment, and evaluation and treatment of periodontal conditions during the second trimester. Patients that agreed in receiving non-surgical periodontal treatment before 28 weeks of pregnancy composed the "intervention group" or IG $(n=16)$. Patients who did not agree in receiving non-surgical periodontal treatment during pregnancy composed the "no intervention group" or NIG $(n=17)$.

Patients included in IG received a non-surgical periodontal treatment consisting of scaling and root planning (SRP), professional prophylaxis (PROPH) and oral hygiene instruction (OHI). Patients included in NIG only received PROPH and $\mathrm{OHI}$ and were referred for caries and periodontal treatment after discharge. Periodontal treatment for those who needed was performed by a single trained periodontist and consisted of mechanical supra and subgingival SRP with Gracey curettes (Hu Friedy, Chicago, IL, USA), PROPH and OHI. Treatment of caries lesions was performed by restorative dentistry specialists at Bauru School of Dentistry, after referral.

\section{Clinical examination}

Patients were evaluated by a previously trained single examiner, different from the one who performed periodontal treatment and unaware of this condition, according to pocket probing depth (PD), clinical attachment level (CAL), sulcus bleeding index (SBI) and plaque index (PI). All patients were examined using good illumination and standardized conditions. PD, CAL and SBI were recorded from four sites per tooth (mesiobuccal, buccal, distobuccal and lingual) with a $15 \mathrm{~mm}$ periodontal probe (Hu-Friedy), except for third molars. The occurrence of bleeding on probing was observed until $15 \mathrm{~s}$ after removal of the probe from sulcus and recorded as present (1) or absent (0), as proposed before ${ }^{1}$. The presence of plaque in the incisal/occlusal, buccal, lingual, mesial and distal aspects of each tooth except for $3^{\text {rd }}$ molars was recorded as ( 1 ) and its absence as $(0)^{24}$. SBI and PI were obtained by the sum of all measurements divided by the number of sites investigated.

\section{Periodontal reevaluation}

Reevaluation of periodontal clinical parameters was performed by a single blinded, previously trained examiner, according to the criteria defined at baseline examination. Reevaluation periods were defined by the consults established by the physician, which took place at the day of delivery and at first post-partum visit, which took place after 7-15 days after birth.

\section{Assessment of pregnancy outcomes}

Primary outcome measures recorded were preterm birth (PTB), defined as delivery $<37$ weeks; low birth weight (LBW), defined as $<2,500$ $\mathrm{kg}$ birth weight; preterm birth and low birth weight (PLBW); late abortion (14-24 gestational weeks); and abortion (<14 gestational weeks). Gestational age was calculated based on the first day of the last period by the physician and nursery staff, followed by confirmation with ultra-sound and physical examinations.

Statistical analysis

Odds ratio (OR) and relative risk (RR) of preterm

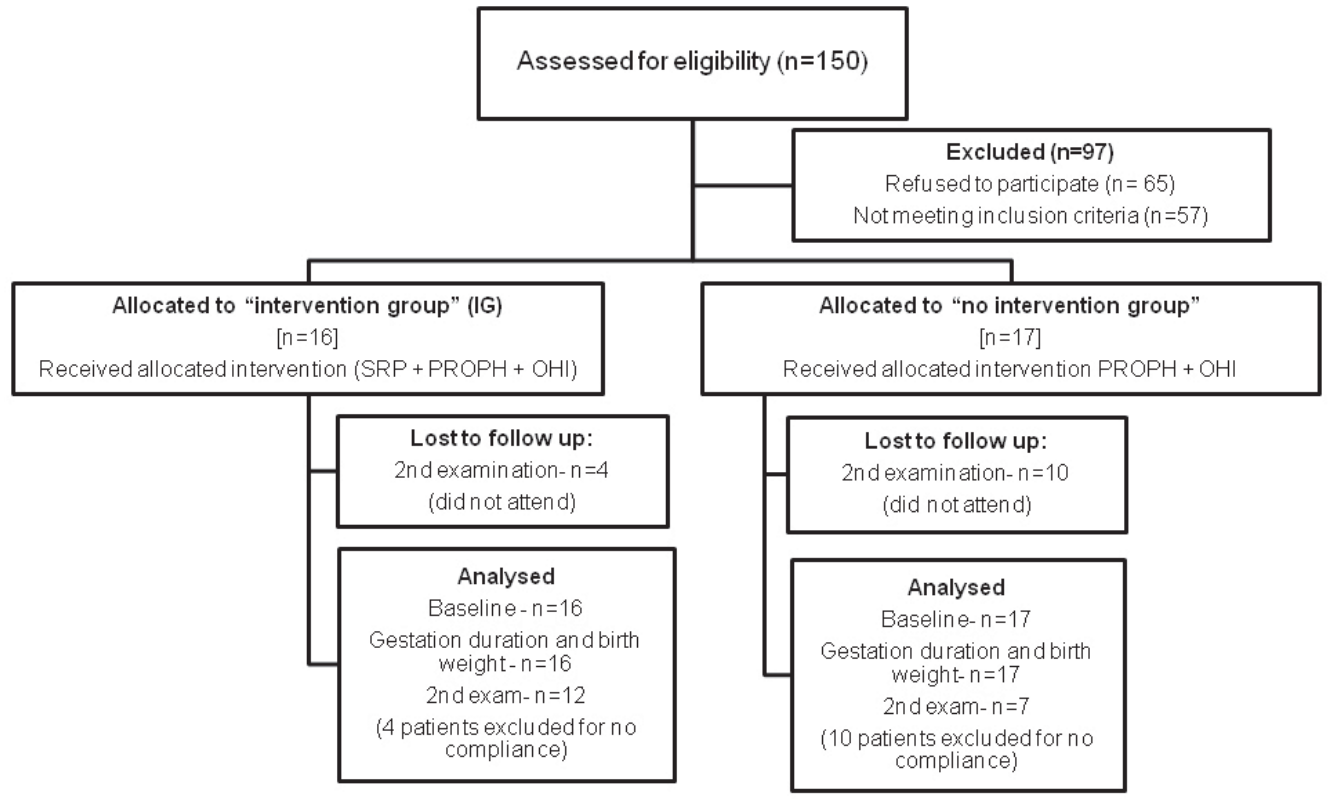

Figure 1- Flow diagram of the progress through the phases of the controlled clinical trial 
( $<37$ weeks), low birth $(<2.5 \mathrm{~kg})$ or preterm and low birth weight in IG and NIG were statistically evaluated using the PACOTICO software (Discipline of Biostatistics, Bauru School of Dentistry, USP, Bauru, SP, Brazil). Comparison of the periodontal conditions between groups at baseline and postoperative examination, birth weight and duration of gestation was performed by unpaired $t$ test for PD and CAL and Mann Whitney for SBI and PI by using GraphPrism 5.0 statistical software (GraphPad
Software, La Jolla, CA, USA), at a 95\% confidence level. Intra-group analysis of treatment effects was performed by paired t-test, at a $95 \%$ confidence level.

\section{RESULTS}

A total of 33 patients were included in the study. IG was composed by 16 patients $(23.75 \pm 4.85$ years) and NIG was composed by 17 patients

Table 1- Social, demographic and overall features of IG (intervention) and NIG (no intervention)

\begin{tabular}{|c|c|c|}
\hline & IG & NIG \\
\hline Age & $23.75 \pm 4.85 \mathrm{yr}$ & $26.00 \pm 6.80 \mathrm{yr}$ \\
\hline Number of missing teeth & $1.5 \pm 2.58$ & $2.41 \pm 3.06$ \\
\hline \multicolumn{3}{|l|}{ Marital status } \\
\hline Married/Stable union & $13(81.25 \%)$ & $13(76.47 \%)$ \\
\hline Single & $0(0 \%)$ & $4(23.53 \%)$ \\
\hline Divorced & $1(6.25 \%)$ & $0(0 \%)$ \\
\hline Other/Non informed & $2(12.5 \%)$ & $0(0 \%)$ \\
\hline \multicolumn{3}{|l|}{ Educational level } \\
\hline None & $0(0 \%)$ & $1(5.88 \%)$ \\
\hline $1^{\text {st }}$ degree (1-9 yr) & $12(75 \%)$ & $10(58.83 \%)$ \\
\hline $2^{\text {nd }}$ degree $(10-12 y r)$ & $2(12.5 \%)$ & $4(23.53 \%)$ \\
\hline $3^{\text {rd }}$ degree $(>13 y r)$ & $2(12.5 \%)$ & $1(5.88 \%)$ \\
\hline Non informed & $0(0 \%)$ & $1(5.88 \%)$ \\
\hline \multicolumn{3}{|l|}{ Professional occupation } \\
\hline Housewife & $10(62.5 \%)$ & $12(70.63 \%)$ \\
\hline Employee & $3(18.75 \%)$ & $2(11.76 \%)$ \\
\hline Autonomous & $0(0 \%)$ & $1(5.85 \%)$ \\
\hline Professor & $2(12.5 \%)$ & $0(0 \%)$ \\
\hline Student & $1(6.25 \%)$ & $2(11.76 \%)$ \\
\hline
\end{tabular}

Table 2- Clinical periodontal parameters of IG (intervention) and NIG (no intervention) at baseline and second examination

\begin{tabular}{|c|c|c|c|c|c|c|c|c|c|}
\hline & & \multicolumn{4}{|c|}{ Baseline } & \multicolumn{4}{|c|}{$2^{\text {nd }}$ exam } \\
\hline & & PD & CAL & SBI & PI & $\mathrm{PD}$ & CAL & SBI & $\mathrm{PI}$ \\
\hline \multirow[t]{3}{*}{ IG } & $\mathrm{n}$ & 1697 & 1697 & 1697 & 1974 & 1151 & 1151 & 1151 & 1591 \\
\hline & mean & $2.10+$ & $0.48 \bullet$ & $0.25 \#$ & 0.71@ & $2.28++$ & $0.56 \bullet$ & $0.29 \# \#$ & 0.71@ \\
\hline & s.d. & 0.02 & 0.02 & 0.01 & 0.01 & 0.02 & 0.02 & 0.01 & 0.01 \\
\hline \multirow[t]{3}{*}{ NIG } & $\mathrm{n}$ & 1741 & 1741 & 1741 & 2062 & 677 & 677 & 677 & 991 \\
\hline & mean & $2.15+$ & $0.47 \bullet$ & $0.26 \#$ & 0.74@ & $2.53++$ & $0.75 \bullet$ & $0.40 \# \#$ & 0.78@ \\
\hline & s.d. & 0.02 & 0.01 & 0.01 & 0.009 & 0.03 & 0.03 & 0.01 & 0.01 \\
\hline IG vs. NIG & $p$ & $0.10^{*}$ & $0.69^{*}$ & $0.48^{* *}$ & $0.07^{* *}$ & $<0.01^{*}$ & $<0.01^{*}$ & $<0.0001^{* *}$ & $<0.055^{* *}$ \\
\hline
\end{tabular}

*Unpaired $t$ test; significant if $p<0.05 .{ }^{* *}$ Mann-Whitney; significant if $p<0.05$. PD- probing depth (in $\mathrm{mm}$ ); CAL- clinical attachment level (in mm); SBI- sulcular bleeding index (absent= 0; present= 1); PI- plaque index; s.d.= standard deviation; $\mathrm{n}=$ number of sites. Equal symbols in rows means no significant differences before (baseline) and after treatment (2nd exam); duplicate symbols in rows means significant differences before (baseline) and after treatment (2nd exam), according to paired $t$ test. 
Table 3- Influence of periodontal treatment on gestation duration and birth weight at IG (intervention) and NIG (no intervention)

\begin{tabular}{lccc}
\hline & IG & NIG & P \\
\hline Gestation duration & $39.19 \pm 0.35$ & $36.31 \pm 1.17$ & $0.02^{*}$ \\
Birth weight & $3.39 \pm 0.11$ & $2.79 \pm 0.20$ & $0.01^{*}$ \\
Odds Ratio & $13.50(\mathrm{Cl}: 1.47-123.75)$ & & $0.02^{*}$ \\
Relative Risk & $7.58(\mathrm{Cl}: 1.07-53.59)$ & & $0.02^{*}$ \\
\hline
\end{tabular}

*unpaired $t$ test; significant if $p<0.05$. Results are mean \pm standard deviation. $\mathrm{Cl}=$ confidence interval.

(26.00 26.80 years). Social and demographic features of IG and NIG are described on Table 1. No statistical differences between groups were observed in age range $(p=0.23)$ and number of missing teeth $(p=0.36)$. Similarly, no significant difference in periodontal conditions at baseline examination was observed between groups, as observed in Table 2.

Treatment of periodontal conditions during the second trimester resulted in stabilization of periodontal parameters at IG, while NIG showed progressive deterioration of periodontal conditions (Table 2). Compliance was low at NIG (41.17\%), while a better compliance was achieved by IG patients $(75 \%)$. Prospective follow-up of patients up to delivery showed that one patient developed preterm birth (36 weeks) in IG, while 6 preterm births ( $<37$ weeks), 1 late abortion ( 24 weeks) and 1 abortion at the first trimester (12 weeks) had developed at NIG. From these, 2 patients had developed very preterm birth (at 29 and 31 weeks).

The mean \pm standard deviation of gestation duration and birth weight at both groups is described on Table 3 . There was a significantly greater gestation duration $(p=0.02)$ and birth weight $(p=0.01)$ at IG than NIG, according to unpaired $t$ test. The risk of developing adverse pregnancy outcomes was significantly increased among patients not submitted to periodontal treatment during pregnancy $(\mathrm{OR}=13.50$; $\mathrm{CI}: 1.47$ 123.75; $p=0.02$ ).

\section{DISCUSSION}

Although periodontal disease has been suggested as an important risk factor for PLBW $2,9,21-23,26,29$, recent studies failed to find this association ${ }^{7,19}$. Some studies associated periodontal disease with adverse pregnancy outcomes, such as late abortion $^{7,27}$ or preeclampsia ${ }^{27}$.

The control of periodontal disease during pregnancy, as in this study, would contribute to a decrease in adverse pregnancy outcomes, which has been established in other large $8,10,12,13$ and small ${ }^{18,26}$ sample population clinical trials already described in the literature, conferring the best risk indicator ${ }^{6}$. However, a very recent study including 757 patients randomly assigned to active treatment (scaling and root planning; $n=378$ ) and control (polishing; $n=379$ ) suggested that non-surgical treatment of periodontal disease during pregnancy did not significantly reduced the risk of spontaneous premature delivery (SPTD) at $<35$ weeks of gestation, but rather increased the risk of SPTD in patients receiving active treatment ${ }^{15}$. Similarly, other reports ${ }^{20}$ in literature have suggested that treatment of periodontal disease during pregnancy, although not hazardous to the mother, does not reduce the rates of premature delivery.

These conflicting results may pose a question if active treatment of periodontal disease during pregnancy is beneficial to both mother and baby, and would really contribute for a decrease in the rates of premature birth and low birth weight. Vettore, et al. ${ }^{28}$ (2006) concluded, in a systematic review on periodontal disease as a risk factor for adverse pregnancy outcomes, that there is a clear heterogeneity between studies concerning measurement of periodontal disease and selection of type of adverse pregnancy outcome. In addition to that, most studies did not control for confounders, thus raising serious doubts about their conclusions.

One possible reason for misleading results in case-control and controlled clinical trials could be attributed to the existence of multiple risk factors for preterm birth or low birth weight, many of which are common to periodontal disease ${ }^{17,29}$. Because of that, it was proposed that large sample population studies should be performed to investigate the influence of periodontal disease in preterm low birth weight, excluding the influence of other recognized risk factors, such as infection of ascending urinary tract or preeclampsia ${ }^{17}$.

A limitation of this study is related to the small sample size. Although 150 patients were initially assessed as eligible, 65 refused to participate and 52 were at the third trimester, when periodontal treatment should not be performed ${ }^{12,13}$. The main reasons for refusal or exclusion were: necessity of taking care of the children $(60 \%)$, getting back to work $(20 \%)$, and fear of being treated during pregnancy or missing scheduled visits (20\%). The allocation of patients to IG or NIG was based on the agreement of patients in receiving nonsurgical periodontal treatment before 28 weeks of pregnancy, after oral information of its importance 
and reading of the informative letter. Those who did not agree in receiving non-surgical periodontal therapy during pregnancy constituted the NIG, meaning that no treatment was performed during pregnancy. Those who agreed in receiving nonsurgical periodontal treatment constituted IG, meaning that periodontal treatment was performed before 28 weeks of gestation. Sadatmansouri, Sedighpoor and Aghalloo ${ }^{26}$ (2006) also conducted a clinical trial in 30 pregnant women from $18-35$ years presenting moderate or severe periodontitis who were randomly assigned to a "no treatment" $(n=15)$ or "treatment" group $(n=15)$, showing a $26.7 \%$ rate of PLBW in untreated patients and no preterm birth or low birth weight at treatment group.

In the antenatal care program attended by the patients, risk factors that could result in preterm birth or low birth weight were under control by an interdisciplinary team, including the physician, dietary consultant, psychologist, and nursery staff. The innovation of our study was introducing a specialized dental treatment during pregnancy, contributing to improve health conditions of the mother. Considering that, the possible influence of periodontal disease in adverse pregnancy outcomes could be evaluated independent from other risk factors, which may be considered as a positive aspect of our study.

When performing small-size clinical trials, the general features of test and control groups should be strictly the same in order to warrant that a specific risk factor is under evaluation without the influence of other recognized variables ${ }^{6,11,17}$, such as maternal height and weight, age $<15$ years or $>39$ years, low economic and educational level, stress, anxiety, excess of physical activity, smoking, alcohol or drugs abuse, absence of antenatal care, number of previous children born alive, number of previous abortion, late abortion or preterm low birth weight. In the present study, general characteristics of the sample were strictly the same at baseline examination, being in agreement with those requisites.

A worsening of periodontal conditions in both treated and untreated groups was observed in this study. However, statistically significant differences were observed at IG in PD and SBI, with no significant changes in CAL and PI. The facts that periodontal conditions of NIG at the second examination were significantly worse than those of IG and had worsened from baseline measurements, suggest that a worsening in periodontal conditions is expected as pregnancy develops ${ }^{14,21}$, especially in low educational and economic level patients ${ }^{14,30}$. However, periodontal care during pregnancy could limit this worsening.

Other large sample population research have also suggested that periodontal treatment result in improvement or stabilization of periodontal conditions and a decrease in PLBW rates ${ }^{8,10,12,13,18}$. Periodontal treatment consisted of non-surgical therapy performed at the second trimester along with mouthwashing with $0.12 \%$ chlorhexidine solution decreased PLBW rates ${ }^{12,13}$, while the association of non-surgical periodontal therapy and metronidazole resulted in no improvement ${ }^{10}$. The absence of periodontal treatment in patients showing inflamed sites during pregnancy, as suggested in the present study, can be considered as a risk factor for adverse pregnancy outcomes $[\mathrm{OR}=13.50, \mathrm{CI}: 1.47-123.75 ; \mathrm{p}=0.02]$, since untreated patients showed a significant worsening of periodontal conditions during pregnancy.

This is especially important if one assumes that periodontal disease progression during pregnancy is strongly associated with adverse pregnancy outcomes ${ }^{21}$. Bleeding on probing reflects ulceration of periodontal pocket epithelium, which could in turn reflect a systemic marker that represents tissue invasion and systemic dissemination of periodontopathic bacteria 4,22,23,25. Furthermore, a temporal relation between exposure measures and outcome should be considered, meaning that clinical attachment level represent cumulative history of periodontal disease of the individual throughout life, influencing development of systemic diseases that requires prolonged exposure to risk factors, such as cardiovascular diseases ${ }^{4}$. On the other hand, probing depth measurements and bleeding on probing reflects current disease status and would be more informative of recent consequences, requiring acute exposition to the risk factor, such as adverse pregnancy outcomes, ${ }^{4,21-23}$. In the present study, IG showed significantly less bleeding sites and shallower periodontal pockets close to delivery or shortly after birth along with a $6.25 \%$ rate of adverse pregnancy outcomes, while NIG showed a $47.05 \%$ rate of adverse pregnancy outcomes, as in other studies $8,10,13,14,18$.

Although these findings seem to be relevant, especially because other risk factors for preterm birth and low birth weight were controlled, larger sample populations should be evaluated in order to establish a treatment protocol for pregnant women. The inclusion of specialized dental treatment during antenatal care should be considered in order to improve health conditions of the mother and the baby.

\section{CONCLUSIONS}

These findings suggest that performing periodontal treatment during the second trimester of gestation would decrease the risk of development of adverse pregnancy outcomes, which could imply that periodontal disease can be considered as a risk 
factor for adverse pregnancy outcomes, especially preterm birth and/or low birth weight.

\section{ACKNOWLEDGEMENTS}

The authors would like to thank Jardim Redentor I Public Health Center for the cooperation with this project. Special thanks to the students Ana Paula Merci, Joanelisa Cantarelli and Cristiani Pereira Abreu for the technical support.

\section{REFERENCES}

1- Ainamo J, Bay I. Problems and proposals for recording gingivitis and plaque. Int Dent J. 1975;25:229-35.

2- Alves RT, Ribeiro RA. Relationship between maternal periodontal disease and birth of preterm low weight babies. Braz Oral Res. 2006;20:318-23.

3- Armitage GC. Periodontal disease and pregnancy: discussion, conclusions and recommendations. Ann Periodontol. 2001;6:18992.

4- Beck JD, Offenbacher S. Relationships among clinical measures of periodontal disease and their association with systemic markers. Ann Periodontol. 2002; 7:79-89.

5- Brasil. Ministério da Saúde. Secretaria de Atenção à Saúde. Departamento de Ações Programáticas Estratégicas. Prenatal and puerpera: humanized and qualified care: techinical manual. Brasília: Ministério da Saúde; 2005.

6- Clothier B, Stringer M, Jeffcoat MK. Periodontal disease and pregnancy outcomes: exposure, risk and intervention. Best Pract Res Clin Obstet Gynaecol. 2007;21:451-66.

7- Farrel S, Ide M, Wilson RF. The relationship between maternal periodontitis, adverse pregnancy outcome and miscarriage in never smokers. J Clin Periodontol. 2006;33:115-20.

8- Gazolla CM, Ribeiro A, Moyses MR, Oliveira LA, Pereira LJ, Sallum AW. Evaluation of the incidence of preterm low birth weight in patients undergoing periodontal therapy. J Periodontol. 2007;78:842-8.

9- Jeffcoat MK, Geurs NC, Reddy MS, Cliver SP, Goldenberg RL, Hauth JC. Periodontal infection and preterm birth: results of a prospective study. J Am Dent Assoc. 2001;132:875-80.

10- Jeffcoat MK, Hauth JC, Geurs NC, Reddy MS, Cliver SP, Hodgkins PM, et al. Periodontal disease and preterm birth: results of a pilot intervention study. J Periodontol. 2003;74:1214-8.

11- Lockwood CJ. Predicting premature delivery - no easy task. N Engl J Med. 2002;346:282-4.

12- López NJ, Silva I, Ipinza J, Gutiérrez J. Periodontal therapy reduces the rate of preterm low birth weight in women with pregnancy-associated gingivitis. J Periodontol. 2005;76(11 Suppl):2144-53.

13- López NJ, Smith PC, Gutiérrez J. Periodontal therapy may reduce the risk of preterm low birth weight in women with periodontal disease: a randomized controlled trial. J Periodontol. 2002;73:911-24.
14- Machuca G, Khoshfeiz O, Lacalle JR, Machuca C, Bullón P. The influence of general health and socio-cultural variables on the periodontal condition of pregnant women. J Periodontol. 1999;70:779-85.

15- Macones GA, Parry S, Nelson DB, Strauss JF, Ludmir J, Cohen AW, et al. Treatment of localized periodontal disease in pregnancy does not reduce the occurrence of preterm birth: results from the Periodontal Infections and Prematurity Study (PIPS). Am J Obstet Gynecol. 2010;202:147; e1-8.

16- Madianos PN, Lieff S, Murtha AP, Boggess KA, Auten RL Jr, Beck $J \mathrm{D}$, et al. Maternal periodontitis and prematurity. Part II: maternal infection and fetal exposure. Ann Periodontol. 2001;6:175-82.

17- Michalowicz BS, Durand S. Maternal periodontal disease and spontaneous preterm birth. Periodontol 2000. 2007;44:103-12.

18- Mitchell-Lewis D, Engebretson SP, Chen J, Lamster IB, Papapanou PN. Periodontal infections and pre-term birth: early findings from a cohort of young minority women in New York. Eur J Oral Sci. 2001;109:34-9.

19- Moore S, Randhawa M, Ide M. A case-control study to investigate an association between adverse pregnancy outcome and periodontal disease. J Clin Periodontol. 2005;32:1-5.

20- Offenbacher S, Beck JD, Jared HL, Mauriello SM, Mendoza LC, Couper DJ, et al. Effects of periodontal therapy on rate of preterm delivery: a randomized controlled trial. Obstet Gynecol. 2009;114:551-9.

21- Offenbacher S, Boggess KA, Murtha AP, Jared HL, Lieff S, McKaig RG, et al. Progressive periodontal disease and the risk of very preterm delivery. Obstet Gynecol. 2006;107:29-36.

22- Offenbacher S, Katz V, Fertik G, Collins J, Boyd D, Maynor G, et al. Periodontal infection as a possible risk factor for preterm low birth weight. J Periodontol. 1996;67(10 Suppl):1103-13.

23- Offenbacher S, Lieff S, Boggess KA, Murtha AP, Madianos PN, Champagne CM, et al. Maternal periodontitis and prematurity. Part I: Obstetric outcome of prematurity and growth restriction. Ann Periodontol. 2001;6:164-74.

24- O 'Leary TJ, Drake RB, Naylor JE. The plaque control record. J Periodontol. 1972;43:38.

25- Romero R, Chiquito CS, Elejalde LE, Bernardoni CB. Relationship between periodontal disease in pregnant women and the nutritional condition of their newborns. J Periodontol. 2002; 73:1177-83.

26- Sadatmansouri S, Sedighpoor N, Aghaloo M. Effects of periodontal treatment phase I on birth term and birth weight. J Indian Soc Pedod Prev Dent. 2006;24:23-6.

27- Siqueira FM, Cota LO, Costa JE, Haddad JP, Lana AM, Costa FO. Intrauterine growth restriction, low birth weight, and preterm birth: adverse pregnancy outcomes and their association with maternal periodontitis. J Periodontol. 2007;78:2266-76.

28- Vettore MV, Lamarca GA, Leão AT, Thomaz FB, Sheiham A, Leal MC. Periodontal infection and adverse pregnancy outcomes: a systematic review of epidemiological studies. Cad Saude Publica. $2006 ; 22: 2041-53$.

29- Williams CE, Davenport ES, Sterne JA, Sivapathasundaram V, Fearne JM, Curtis MA. Mechanisms of risk in preterm lowbirthweight infants. Periodontology 2000. 2000;23:142-50.

30- Yalcin F, Eskinazi E, Soydinc M, Basegmez C, Issever H, Isik G, et al. The effect of sociocultural status on periodontal conditions in pregnancy. J Periodontol. 2002;73:178-82. 